\title{
A COMPLETE SAMPLE OF LONG BRIGHT SWIFT GRBS
}

\author{
G. Tagliaferri ${ }^{1}$, R. Salvaterra ${ }^{2}$, S. Campana ${ }^{1}$, S. Covino $^{1}$, P. D'Avanzo ${ }^{1}$, \\ D. Fugazza ${ }^{1}$, G. Ghirlanda ${ }^{1}$, G. Ghisellini ${ }^{1}$, A. Melandri ${ }^{1}$, B. Sbarufatti ${ }^{1}$, \\ S. Vergani ${ }^{1}$ and L. Nava ${ }^{3}$
}

\begin{abstract}
Starting from the Swift sample we defined a complete subsample of 58 bright long Gamma Ray Bursts (GRB), 52 of them (90\%) with a redshift determination, in order to characterize their properties. This complete sample of bright long- GRBs allowed us to investigate their evolution with cosmic time and properties. We focused in particular on the GRB luminosity function, on the spectral-energy correlations of their prompt emission, on the nature of dark bursts and on possible correlations between the prompt and the X-ray afterglow properties.
\end{abstract}

\section{Introduction}

Long Gamma Ray Bursts (GRB) are a powerful alternative possibility to study the star formation rate and probably the only one to directly study the stellar evolution up to the very young Universe, soon after the first massive stars have started to form and die. In fact, GRBs are associated to the death of very massive stars and, therefore, with star forming regions. Moreover, they are very bright objects that can be detected up to extremely high redshifts: so far we have a secure spectroscopic redshift of $z=8.2$ and a photometric record holder of $z=9.4$. At these redshift, the Universe was very young, less than $10 \%$ of its current age. Hence, although it is still not completely clear if GRBs provide un unbiased view, they can be used to study the history of the star formation and of the metallicity enrichment from the local Universe up to the epoch where the first stars are thought to form.

About one third of the bursts detected by Swift (Gehrels et al. 2004) has a measured redshift. While this represents an enormous improvement with respect to the pre-Swift situation, this is still to low to provide a complete sample in

\footnotetext{
1 INAF-OABr, via Bianchi 46, 23807 Merate, Italy;

e-mail: gianpiero.tagliaferri@brera.inaf .it

2 INAF-IASF Milano, via Bassini 15, 20133 Milano, Italy

3 APC Université Paris Diderot, 10 rue Domon et Duquet, 75205 Paris, France
} 

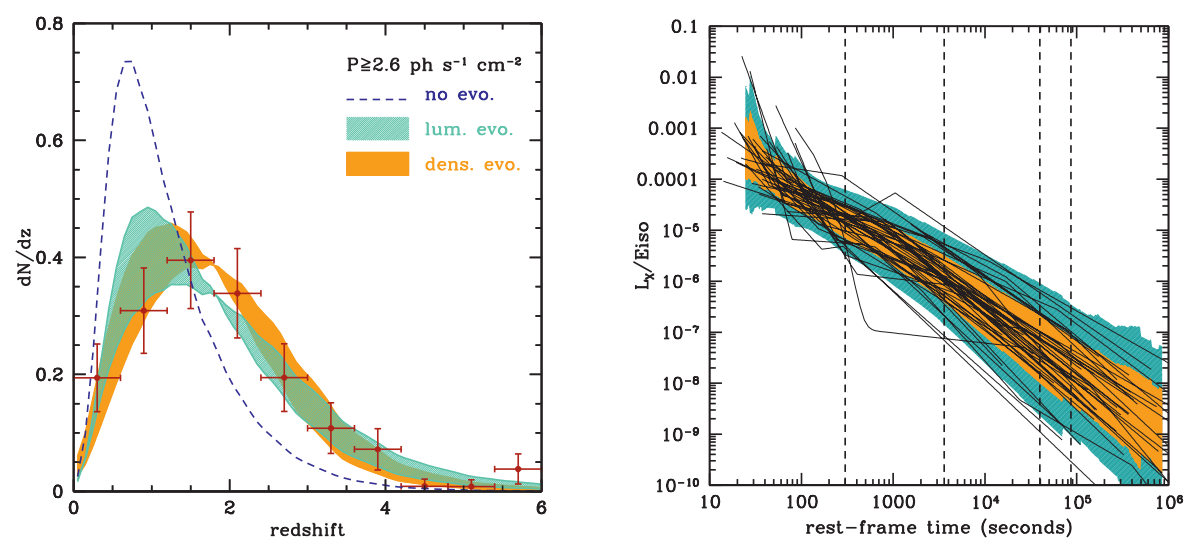

Fig. 1. Left panel: normalized redshift distribution of GRBs with $P \geq 2.6 \mathrm{ph} \mathrm{s}^{-1}$ $\mathrm{cm}^{-2}$ in the band $15-150 \mathrm{keV}$. Data points show the observed redshift distribution. The dashed line shows the expected distribution for the no-evolution case. Results of luminosity and density evolution models are also shown. Right panel: best fit of the X-ray luminosity light curves normalized to their $E_{\text {iso }}$. The rest frame times at which we computed the various correlations (see text) are marked with vertical dashed lines. The light (dark) shaded area represents the $1 \sigma(2 \sigma)$ scatter around the mean value of the $L_{i s o} / E_{i s o}$ distribution (see D'Avanzo et al. 2012 for more details).

redshift. Moreover, this sample is very heterogeneous. Therefore we started from the criteria proposed by Jakobsson et al. (2004): i) the burst has been well localized by Swift/XRT and its coordinate quickly distributed; ii) the Galactic extinction in the burst direction is low $\left(A_{V}<0.5\right)$; iii) the GRB declination is $-70^{\circ}<\delta<70^{\circ}$; iv) the Sun-to-field distance is $\theta_{\text {Sun }}>55^{\circ}$; v) no nearby bright stars are present. This increases the completeness level in redshift to $\sim 50 \%$. We then restricted our sample to GRBs that are relatively bright in the $15-150 \mathrm{keV}$ Swift/BAT band, i.e. with a 1-s peak photon flux $P \geq 2.6 \mathrm{ph} \mathrm{s}^{-1} \mathrm{~cm}^{-2}$. This corresponds to an instrument that is $\sim 6$ times less sensitive than Swift, which give us a high level of confidence that all GRBs with a flux higher then our limit and that are inside the FOV of BAT when they explode will be detected. Therefore, our sample is complete with respect to our selection criteria and provide an unbiased view of the bright end of the GRB Log N - Log S. Clearly, we can not say what are the property of the burst at lower fluxes, to this end other samples are better suited. It also provide a sample that has more than $90 \%$ completeness in redshift. In fact, up to the end of May 2011, 58 GRBs match our selection criteria (see Salvaterra et al. 2012) and 52 of them have a measured redshift. Moreover, for 3 of the other 6 bursts the afterglow or the host galaxy has been detected in at least one optical filter, so that $\sim 95 \%$ of the bursts in our sample have a constrained redshift. We note that, while our sample represents only $\sim 10 \%$ of the full Swift sample, it contains more than $30 \%$ of long GRBs with known redshift. 


\section{Luminosity function, spectral-energy correlations, dark bursts}

Our complete Swift sample is very powerful to test the evolution of the long GRB population with redshift. Still the number of burst is not large enough to derive the present day GRB rate density and obtain meaningful constrains to the GRB luminosity function (LF). In fact, with a sample of 58 burst it is not possible to discriminate between different evolution scenarios for the LF, the error bars are so large that even a no-evolution scenario could reproduce the data. Therefore, we derived the GRB luminosity function (LF) by jointly fitting the observed differential number counts in the $50-300 \mathrm{keV}$ band of BATSE (Stern et al. 2002) and the observed redshift distribution of bursts in our sample. We note that the best-fit parameters derived in this way provide a good fit also of the Swift differential peak-flux number counts once the $15-150 \mathrm{keV}$ band, the field of view of $1.4 \mathrm{sr}$ and the observing lifetime of Swift are considered. We explored two general expressions for the GRB LF: a single power-law with an exponential cut-off at low luminosity and a broken power-law LF (Salvaterra et al. 2012).

As shown in Figure 1, left panel, if we assume that long GRBs trace the cosmic star formation and that their LF is constant in redshift, i.e. the no-evolution scenario, we can not reproduce the observed redshift distribution of our sample, confirming previous findings (Kistler et al. 2009; Virgili et al. 2011; Robertson \& Ellis 2012; Jakobsson et al. 2012). We note that Elliott et al. (2012), using a different complete sample of GRBs observed by GROND of size (39 objects) similar to our sample, claim that there is no need of evolution in the GRB luminosity function. As said above, with a small number of sources the error bars in the data points are too big and even a non-evolving luminosity function is consistent with the data. We find the same results if we use only our sample of 58 sources, this is why we choose to fit also the BATSE sample. Therefore, to fit our data we considered two evolution scenarios: i) a luminosity evolution model in which the GRBs at higher redshift are typically brighter than the bursts at lower redshift and, ii) a density evolution model, in which the GRB formation rate increases with redshift. Both models can reproduce the observed redshift distribution; in particular we find that either the typical burst luminosity increases as $(1+z)^{2.3 \pm 0.6}$ or the GRB rate density as $(1+z)^{1.7 \pm 0.5}$ on top of the known cosmic evolution of the SFR (as computed by Li 2008). These result do not depend on the assumed expression of the GRB LF. We can also reproduce our data assuming no evolution of the GRB LF if we assume that GRBs form preferentially in low-metallicity environments. In this case we find that the metallicity threshold for GRB formation should be lower than $0.3 Z_{\odot}$ in order to account for the observations (Salvaterra et al. 2012).

One of the most debated issue in the GRB field is the existence or otherwise of the correlations between the GRBs spectral peak energy $E_{\text {peak }}$, the isotropic energetics $E_{\text {iso }}$ and the isotropic luminosity $L_{\text {iso }}$. Having a complete sample of bright long GRBs, we can properly investigate about these correlations. As shown by Nava et al. (2012) a strong correlation is found between $E_{\text {peak }}-E_{\text {iso }}$ and $E_{\text {peak }}-$ $L_{\text {iso }}$ for the bursts of this complete sample, with only one outlier, GRB 061021. Their slopes, normalizations and dispersions are consistent with those found with 
the whole sample of bursts with measured redshift and $E_{\text {peak }}$. The biases present in the total sample commonly used to study these correlations do not affect their properties. We also find that there is no evolution with redshift of the $E_{\text {peak }}-E_{\text {iso }}$ and $E_{\text {peak }}-L_{\text {iso }}$ correlations. One of the argument used against these correlation is that they are due to the presence of flux limits in the existing samples of GRBs. By performing Monte Carlo simulations under different assumptions for their LF we studied the possible effects caused by the flux-limit selection in our complete sample on the $E_{\text {peak }}-L_{\text {iso }}$ correlation. If we assume that this correlation does not exist, we are unable to reproduce it as due to the flux limit threshold of our complete sample. The null hypothesis can be rejected at more than $2.7 \sigma$ level of confidence (Ghirlanda et al. 2012a). We used this complete sample, together with other samples, also to study the distributions of the GRB jet opening angle $\theta_{j e t}$ and the bulk Lorentz factor $\Gamma_{0}$. We find that on average the "faster" bursts, i.e. those with larger values of $\Gamma_{0}$, have smaller values of $\theta_{j e t}$ (Ghirlanda et al. 2012b).

Beside studying the properties of the GRB prompt emission, our complete sample can be used to statistically study also their afterglow properties. It is well known that while thanks to its prompt reaction Swift almost always find a X-ray afterglow associated to a GRB, in the optical/NIR this is not the case. These GRBs are called "dark"-GRBs, for which there are various definitions based on the ratio between the optical and the X-ray fluxes (or their upper limits). With our complete sample of bright GRBs we have established the existence of a genuine dark population with $\sim 30 \%$ of dark-burst (according to the definition of Jakobsson et al. 2004) expected for the whole class of long GRBs (Melandri et al. 2012). This population of dark-bursts has a redshift distribution and prompt properties very similar to those of the whole sample. At the same time their de-absorbed X-ray flux is slightly higher than the one of the non-dark events, while their optical flux is at the lower tail of the optical flux distribution. All these properties suggest that dark-bursts events generate in much denser environments with respect to normal bright events. In agreement with previous results, we can therefore exclude the high- $z$ and the low-density scenarios as the cause of their darkness. The major cause of the optically dark events is the dust extinction (Melandri et al. 2012). We also find that there is a very tight correlation between the GRB darkness and their high X-ray column densities (Campana et al. 2012; Fynbo et al. 2009). Again, a strong indication that dark-GRBs are formed in a metal-rich environment where dust must be present. For the full sample of X-ray afterglows, using the Swift $\mathrm{X}$-ray Telescope data, we find that the distribution of their intrinsic absorbing $\mathrm{X}$-ray column densities has a mean value of $\log \left(N_{H} / \mathrm{cm}^{-2}\right)=21.7 \pm 0.5$, consistent with the one derived from the total sample of GRBs with redshift. The observed mild increase of the intrinsic column density with redshift is probably due to the contribution of intervening systems along the line of sight. (Campana et al. 2012).

To investigate whether there is a correlation between the X-ray afterglow luminosity and the prompt emission properties we computed the afterglow X-ray luminosities at four different rest frame times $(5 \mathrm{~min}, 1 \mathrm{hr}, 11 \mathrm{hr}$ and $24 \mathrm{hr}$ after trigger). Indeed, we find that $E_{i s o}, L_{i s o}$ and the rest frame peak energy $E_{\text {peak }}$ do correlate with the rest frame afterglow $\mathrm{X}$-ray luminosity, but the significance 
of each correlation decreases over time. This result can be explained by a GRB $\mathrm{X}$-ray light curve due to a combination of different components whose relative contribution and weight change with time, with the prompt and afterglow emission dominating at early and late time, respectively. In particular, we found evidence that the plateau and the shallow decay phase often observed in GRB X-ray light curves are powered by activity from the central engine. The existence of the $L_{X}-E_{\text {iso }}$ correlation at late times (see Fig. 1, right panel) suggests a similar radiative efficiency among different bursts with on average about $6 \%$ of the total kinetic energy powering the prompt emission (D'Avanzo et al. 2012).

\subsection{Conclusions}

Using a well defined complete sample of bright GRBs with more than $90 \%$ of redshift determination, we characterized their luminosity function, properties of their prompt emissions and their correlation with the X-ray afterglow emissions. We find that strong luminosity or density evolution is required in order to reproduce the data. Alternatively, the GRB must preferentially form in low metallicity environment $\left(\leq 0.3 Z_{\odot}\right)$. We confirm the existence of the $E_{\text {peak }}-E_{\text {iso }}$ and $E_{\text {peak }}-L_{\text {iso }}$ correlations, and showed that this can not be due to a bias introduced by the flux limited threshold of our sample. We also find that the cause of the dark nature of some GRBs ( $\sim 30 \%$ of the sample) is most likely due to dust extinction and find a strong correlation between the GRB darkness and their high X-ray column densities. Finally, the afterglow X-ray emissions do correlate with the prompt emissions even at later time (11 hours rest frame), with the X-ray light curve that is powered by activity from the central engine for long times.

\section{References}

Campana, S., Salvaterra, R., Melandri, A., et al., 2012, MNRAS, 421, 1697

D'Avanzo, P., Salvaterra, R., Sbarufatti, B., et al., 2012, MNRAS, 425, 506

Elliott, J., Greiner, J., Khochfar, et al., 2012, A\&A, 539, 113

Fynbo, J.P.U., Jakobsson, P., Prochaska, J.X., et al., 2009, ApJS, 185, 526

Gehrels, N., Chincarini, G., Giommi, P., et al., 2004, ApJ, 611, 1005

Ghirlanda, G., Ghisellini, G., Nava, L., et al., 2012, MNRAS, 422, 2553

Ghirlanda, G., Ghisellini, G., Salvaterra, R., et al., 2012, MNRAS, 428, 1410

Jakobsson, P., Hjorth, J., Fynbo, J.P., et al., 2006, A\&A, 617, L21

Jakobsson, P., Hjorth, J., Malesani, D., et al., 2012, ApJ, 752, 62

Kistler, M.D., Yüksel, H., Beacon, J.F., et al., 2009, ApJ, 705, L104

Li, L.-X., 2008, MNRAS, 388, 1487

Melandri, A., Sbarufatti, B., D'Avanzo, P., et al., 2012, MNRAS, 421, 1265

Nava, L., Salvaterra, R., Ghirlanda, G., et al., 2012, MNRAS, 421, 1256

Robertson, B.E., \& Ellis, R.S., 2012, ApJ, 744, 95

Salvaterra, R., Campana, S., Vergani, S.D., et al., 2012, ApJ, 749, 68

Stern, B.E., Atteia, J.-L., \& Hurley, K., 2002, ApJ, 578, 304

Virgili, F.J., Zhang, B., Nagamine, K., \& Choi, J.-H., 2011, MNRAS, 417, 3025 
